\title{
Interleukin 6, Matrix Metalloproteinase-8 and Tissue Inhibitor-1 Levels in Obesity
}

\author{
Mariana Alves ${ }^{1}$, Ana Carolina Lopes ${ }^{1}$, Jéssica Pontes ${ }^{1}$, Nuno Dias ${ }^{2}$, João Paulo Figueiredo ${ }^{3}$, Nádia \\ Osório ${ }^{1}$, Mário Monteiro ${ }^{2}$ and Armando Caseiro*1 \\ ${ }^{1}$ Polytechnic Institute of Coimbra, ESTeSC - Coimbra Health School, Biomedical Laboratory Sciences, Portugal \\ ${ }^{2}$ Polytechnic Institute of Coimbra, ESTeSC - Coimbra Health School, Medical Image and Radiotherapy, Portugal \\ ${ }^{3}$ Polytechnic Institute of Coimbra, ESTeSC - Coimbra Health School, Complementary Sciences, Portugal \\ *Corresponding author: Armando José Cerejo Caseiro, Department of Biomedical Laboratory Sciences, Polytechnic Institute of \\ Coimbra, Coimbra Health School, Portugal
}

\section{ARTICLE INFO}

Received: 幽 February 05, 2020

Published: 幽 February 13, 2020

Citation: Mariana Alves, Ana Carolina Lopes, Jéssica Pontes, Nuno Dias, João Paulo Figueiredo, Nádia Osório, Mário Monteiro, Armando Caseiro. Interleukin 6, Matrix Metalloproteinase-8 and Tissue Inhibitor-1 Levels in Obesity. Biomed J Sci \& Tech Res 25(4)-2020. BJSTR. MS.ID.004229.

Keywords: Obesity; Inflammation; IL-6; MMP-8; TIMP-1

Abbreviations: ILs: Interleukins; MMPs: Matrix Metalloproteinases; ECM: Extracellular Matrix; CRP: C-Reactive Protein; DEXA: Dual-Energy X-ray Absorptiometry; ASAT: Aspartate Aminotransferase; HDL: High Density Lipoprotein Cholesterol; LDL: Low Density Lipoprotein Cholesterol; OD: Optical Density

\section{ABSTRACT}

Obesity is a nutritional disease characterized by excess of adipose tissue. It is recognized as a low-grade chronic inflammation state characterized by increased serum levels of acute phase proteins such as interleukins (ILs) and cytokines. IL-6 is a proinflammatory cytokine, which is involved in the regulation of lipid metabolism associated with obesity and inflammation. It plays numerous proinflammatory roles. However, IL-6 may also play an anti-inflammatory role. Matrix metalloproteinases (MMPs) participate in several physiological processes such as remodeling of extracellular matrix (ECM), healing, angiogenesis and apoptosis. They are regulated by specific tissue inhibitors (TIMPs) which block access to the active site of MMPs. A dual role for MMP-8 has been described under specific conditions: MMP-8 plays a role in the development of the inflammatory response but appears to play an anti-inflammatory role during recovery and may be crucial for this process.

Aims: To determine the IL-6, MMP-8 and TIMP-1 levels and compare the results between 3 groups of individuals (between 26 and 65 years): normal weight $(n=8)$, overweight $(n=11)$ and obese $(n=22)$.

Material and Methods: The semi-quantification of IL-6, MMP-8 and TIMP-1 was performed through the slot blot technique.

Results: The levels of MMP-8 and IL-6 were higher in the group of overweight individuals than in the normal weight group and in the obese group, but the differences were not statistically significant. TIMP-1 levels were slightly elevated in the group of normal weight individuals and in the group of obese individuals, in relation to the group of overweight individuals, however the differences were not statistically significant. There was also a moderate positive correlation between glycemic levels and the percentage of visceral fat mass $(p<0.0001)$ and between serum levels of MMP-8 and serum levels of IL-6 $(\mathrm{p}<0.05)$.

Discussion and Conclusion: The significant correlation between IL-6 levels and the percentage of visceral fat mass may be related to the fact that adipose tissue is endocrine and produce numerous factors contributing to systemic inflammation. The moderate positive correlation between serum MMP-8 levels and serum IL-6 levels may indicate that these can be stimulated by the same mechanisms in the inflammation process. 


\section{Introduction}

Obesity is a nutritional disorder characterized by excess of adipose tissue. The development of this disorder is associated with coordinated cellular processes, including adipocyte hypertrophy followed by recruitment of adipocyte precursors, and new fat cell differentiation. These processes are also accompanied by neovascularization, which is essential for generation and function of the tissue [1]. The development of obesity involves processes like adipogenesis and extracellular matrix (ECM) remodeling [2]. This disorder is recognized as a low-grade chronic inflammatory state, characterized by the increase of serum levels of acute phase proteins, such as C-reactive protein (CRP), and other interleukins and cytokines [3]. Evidences support that many co-morbidities of obesity, including type 2 diabetes mellitus, nonalcoholic fatty liver disease, steatohepatitis, asthma, cancer, cardiovascular diseases and neurodegenerative diseases, are related to the development of this low-grade chronic inflammation [4]. The primary cause of inflammation induced by obesity is not yet fully understood. The rapid expansion of the adipose tissue may produce intrinsic signals which might trigger an inflammatory response.

These signals include adipocyte death, hypoxia and mechanotransduction resulting from cells and ECM interactions [4]. Another explanation for this trigger may be endoplasmic reticulum stress [4-6]. Adipocyte hyperplasia and hypertrophy may cause mitochondrial and endoplasmic stress, which during fat overload releases additional inflammatory cytokines that can attract macrophages. During adipocyte hypertrophy the expression of Tumor Necrosis Factor $\alpha$, Interleukin 6 (IL-6), Interleukin $1 \beta$ and Prostaglandin E2 are induced, adipocyte die, and neutrophils, monocytes and $\mathrm{T}$ cells are consistently activated [5]. Out of all cytokines deregulated in obesity, IL-6 is one of the most common. The high levels of this interleukin are consistently seen in obese rats and humans [7]. IL-6 is a proinflammatory cytokine, produced by several types of cells, including bone marrow cells and hepatocytes. Some of the proinflammatory roles of this cytokine are B and $\mathrm{T}$ lymphocyte activation, growth and differentiation [8]. However, IL-6 may also have an anti-inflammatory role [9]. This cytokine is involved in the regulation of lipid metabolism associated with obesity and inflammation [10].

Matrix metalloproteinases (MMPs) are zinc and calcium dependent endopeptidases, which participate in several physiological processes such as ECM remodeling, wound repair, angiogenesis and apoptosis. MMPs are able to increase matrix plasticity, enabling adipose tissue remodeling and/or adipocyte hypertrophy [1]. They are secreted by various types of cells, such as inflammatory, neoplastic and epithelial cells, in the form of proenzymes, which require activation by proteolysis. This process is controlled by MMPs specific tissue inhibitors (TIMPs) [11]. TIMPs block access to the MMPs activation site. There are 4 known TIMPs (TIMP -1, -2, -3 e -4), that share similar structural characteristics and have an inhibitory specificity capable of inhibiting most of human MMPs [12]. ECM degradation, in addition to enabling cell migration, also leads to the release of signaling molecules such as chemokines, cytokines and growth factors. It has become widely accepted that MMPs play a central role in the direct activation of signaling molecules, which shows that MMPs also contribute to various aspects of immunity [13].

Recent data suggest that MMPs play an important role in inflammation and the regulation of the immune response. MMPs have been proposed as inflammation regulators, through proteolysis of chemokines, growth factors, receptors and their binding proteins, proteases and protease inhibitors, as well as intracellular multifunctional proteins, resulting in pro- or anti-inflammatory functions leading to tissue homeostasis or to pathology [14]. MMP-8, also known as collagenase- 2 or neutrophils collagenase, plays different roles in regulating of inflammatory response. This MMP is quickly released when neutrophils are activated, in order to ensure their availability in inflammatory sites. A dual function has been described for MMP-8 under specific conditions: it plays a role in the development of the inflammatory response, but it seems to play an anti-inflammatory role during recovery and seems to be crucial to this process [13]. MMP-8 deficient mice showed delayed in the beginning and a slow clearance of local inflammatory infiltrates. Several immunological mediators, such as Macrophage Inflammatory Protein $1 \alpha$, Interleukin 10 and LipopolysaccharideInduced Cytokine, were involved in that characteristic pattern and showed to be substrates of MMP-8 [15].

\section{Material and Methods}

\section{Study Population}

The individuals participating in this study are aged between 26 and 65 years. They were grouped according to their percentage of fat mass in standard weighted individuals (female: $<33 \%$; male: 8-19\%), overweight individuals (female: $\geq 33-39 \%$; male: $\geq 19-25 \%$ ), and obese individuals (female: $>39 \%$; male: $>25 \%$ ) [16]. All participants in this study were non-smokers and had no symptoms of inflammation or any other chronic disease. The percentage of total fat mass and abdominal fat was determined by Dual-Energy X-ray Absorptiometry (DEXA). DEXA's procedure consisted in positioning the patient in General Eletrics equipment, model Lunar iDXA encore 2011, in supine position, within the field defined by the equipment, around 6 to 12 minutes. The individuals, while still, were submitted to low energy X-rays. After attenuation of the X-rays, and their capture by the equipment detectors, the body composition values were obtained. Blood samples were also collected to the participants, obtaining a total of 41 samples: 8 samples of standard weighted individuals, 11 samples of overweight individuals and 22 samples of obese individuals. The data of the study population (individual characteristics, body composition and biochemical markers) are shown on Table 1. The blood collection was carried out in Biomedical Laboratory Science's laboratory, Then, the samples were processed and stored in the freezer at $-80^{\circ} \mathrm{C}$ until analysis. This study respects the principles of the Helsinki declaration and all participants gave their informed consent. 
Table 1: Characterization of the study population.

\begin{tabular}{|c|c|c|c|}
\hline & Standard Weight & Overweight & Obese \\
\hline \multicolumn{4}{|c|}{ Individual Characteristics } \\
\hline Genre (n) & \multirow{2}{*}{$7(87,50 \%)$} & \multirow{2}{*}{$9(81,82 \%)$} & \multirow{2}{*}{$11(50,00 \%)$} \\
\hline Female & & & \\
\hline Male & $1(12,50 \%)$ & $2(18,18 \%)$ & $11(40,00 \%)$ \\
\hline Age (years) & $40,88 \pm 9,48$ & $48,00 \pm 10,50$ & $46,95 \pm 9,61$ \\
\hline \multicolumn{4}{|c|}{ Body Composition } \\
\hline Total fat mass percentage (\%) & $26,70 \pm 5,288$ & $33,65 \pm 5,629$ & $38,46 \pm 7,149 ¥$ \\
\hline Visceral fat mass percentage (\%) & $18,73 \pm 5,875$ & $33,44 \pm 6,180$ & $43,77 \pm 8,017 \S ¥$ \\
\hline \multicolumn{4}{|c|}{ Biochemical Markers (mg/dl) } \\
\hline Fasting glycemia & $81,40 \pm 6,43$ & $87,40 \pm 7,89$ & $94,26 \pm 14,66 \#$ \\
\hline Total cholesterol & $187,40 \pm 28,65$ & $203,00 \pm 28,65$ & $190,20 \pm 37,14$ \\
\hline HDL cholesterol & $62,10 \pm 13,84$ & $62,00 \pm 18,70$ & $53,88 \pm 16,10$ \\
\hline LDL cholesterol & $107,30 \pm 23,27$ & $126,40 \pm 33,30$ & $117,50 \pm 35,48$ \\
\hline Triglycerides & $77,73 \pm 36,10$ & $73,08 \pm 15,15$ & $94,30 \pm 60,36$ \\
\hline Albumin & $4,473 \pm 0,2501$ & $4,511 \pm 0,3274$ & $4,501 \pm 0,2433$ \\
\hline Total proteins & $6,579 \pm 0,3639$ & $6,615 \pm 0,3935$ & $6,524 \pm 0,2642$ \\
\hline ALAT & $16,83 \pm 8,263$ & $24,54 \pm 13,68$ & $29,95 \pm 15,51 ¥$ \\
\hline ASAT & $17,86 \pm 3,574$ & $19,88 \pm 5,265$ & $19,65 \pm 6,501$ \\
\hline Creatinine & $0,6788 \pm 0,1766$ & $0,6436 \pm 0,1428$ & $0,9057 \pm 0,8386$ \\
\hline Urea & $34,41 \pm 8,185$ & $33,36 \pm 6,751$ & $34,66 \pm 10,02$ \\
\hline
\end{tabular}

Note: The data for the individual characteristics are presented as mean \pm standard deviation;

( ${ }^{*} \mathrm{p} \leq 0,05 ;{ }^{* *} \mathrm{p} \leq 0,01 ;{ }^{* * *} \mathrm{p} \leq 0,001 ; \S={ }^{*}$ overweight vs obese; \# = ** standard weight vs obese; $¥={ }^{* * *}$ standard weight vs obese.

\section{Sample Treatment}

Biochemical Characterization of the Population: The measurement of glucose, total cholesterol, high density lipoprotein cholesterol (HDL), low density lipoprotein cholesterol (LDL), triglycerides, albumin, creatinine, alanine aminotransferase (ALAT), aspartate aminotransferase (ASAT), total proteins and urea was determined in the automated clinical analyzer Prestige 24i, using Cormay kits: Prestige 24i GLU, Prestige 24i CHOL, Prestige 24i HDL Direct, Prestige 24i TG, Prestige 24i ALBUMIN, Prestige 24i CREATININE, Prestige 24i ALAT, Prestige 24i ASAT, LQ total Protein, Prestige 24i UREA (PZ Cormay S. A., Poland).

Slot-Blot: Slot-blot analysis was carried out according to Caseiro, et al. [17]. The serum samples were diluted on a buffered saline solution with Tris (TBS) (10 mM Tris, $200 \mathrm{mM} \mathrm{NaCl),} \mathrm{with} \mathrm{the}$ objective of obtaining a final protein concentration of $0,02 \mu \mathrm{g} / \mu \mathrm{L}$ and applying a volume of $100 \mu \mathrm{L}$, except in samples to semi-quantify IL-6, in which was used a final protein concentration of $0,04 \mu \mathrm{g} / \mu \mathrm{L}$. Nitrocellulose membranes - Hybond ECL Nitrocellulose Membrane (GE Healthcare, Pittsburgh, USA) were blocked with a $5 \%(\mathrm{~m} / \mathrm{v})$ solution of skimmed milk powder in TBS-Tween (TBS-T). They were then incubated for one and a half hours at room temperature with constant shaking, with primary antibody anti-MMP-8 (Clone 100619 MAB 9021; from R\&D systems, Minneapolis, USA) or primary antibody anti-IL6 (ab9324, from abcam, Cambridge, UK), or primary antibody anti-TIMP-1 (Clone 63515; MAB 970 from R\&D systems, Minneapolis, USA), diluted to 1:1000.
Afterwards, all membranes were washed three times, for 10, 15 and 10 minutes, respectively, with TBS-T and incubated for an hour and a half with secondary antibody (Horseradish Peroxidase conjugated goat anti mouse, GE Healthcare, Pittsburgh, UK), at a dilution of 1:1000. The detection was performed using Amersham ECL start Western Blotting Detection Reagent kit (GE Healthcare, Pittsburgh, $U K)$ and exposed to a photographic film Kodak BioMax Light Film (Carestream Health, Rocherter, USA) in a cassette (Kodak ${ }^{\circledR}$ X-OMAT Casset, Carestream Health, Rocherter, USA) for 3 minutes. The analysis of the optical density of the bands was carried out using the image acquisition system GELDOC ${ }^{T M} X R^{+}$(Bio-Rad Hercules, USA) and the software ImageLab ${ }^{\circledR}$ Verion 3.0 (Bio-Rad Hercules, USA).

Statistic: Statistical calculations were performed using GraphPad Prism version 5.0 for Windows (GraphPad Software, San Diego, California, EUA). The normality of data distribution was assessed using the test Shapiro-Wilk. Values are presented as mean \pm standard deviation and values for optical density (OD) are expressed in arbitrary units. Regarding the differences between the optical density measurements of the sample groups, they were determined using the ANOVA - 1 Factor and Kruskall Wallis. The correlation between variables was assessed using the correlation coefficient Rho of Spearman or R of Pearson, depending on the normality of the results obtained. The differences/correlation between the groups were considered statistically significant, when a random error $\mathrm{p} \leq$ 0,05 was assumed with a confidence level of $95 \%$. 


\section{Results}

Regarding blood glucose, higher levels were found in the group of obese individuals $(94,26 \pm 14,66 \mathrm{mg} / \mathrm{dl})$ when compared to the group of overweight individuals $(87,55 \pm 7,889 \mathrm{mg} / \mathrm{dl})$ and standard weighted individuals $(81,40 \pm 6,433 \mathrm{mg} / \mathrm{dl})$. There were statistically significant differences between the groups of obese and standard weighted individuals $(\mathrm{p} \leq 0,01)$ (Figure 1$)$. The MMP-8 levels were higher in the group of overweight individuals (13350 \pm 8273 ), in relation to the group of standard weighted individuals (9856 \pm 7773 ) and the group of obese individuals (9586 \pm 4192 ), however the differences did not present statistical significance (Figure 2). The IL-6 levels were slightly higher in the group of overweight individuals (84329 \pm 35718 ), when compared to the group of standard weighted individuals $(73872 \pm 44865)$ and the group of obese individuals (76005 \pm 24852$)$, however the differences did not present statistical significance (Figure 3).

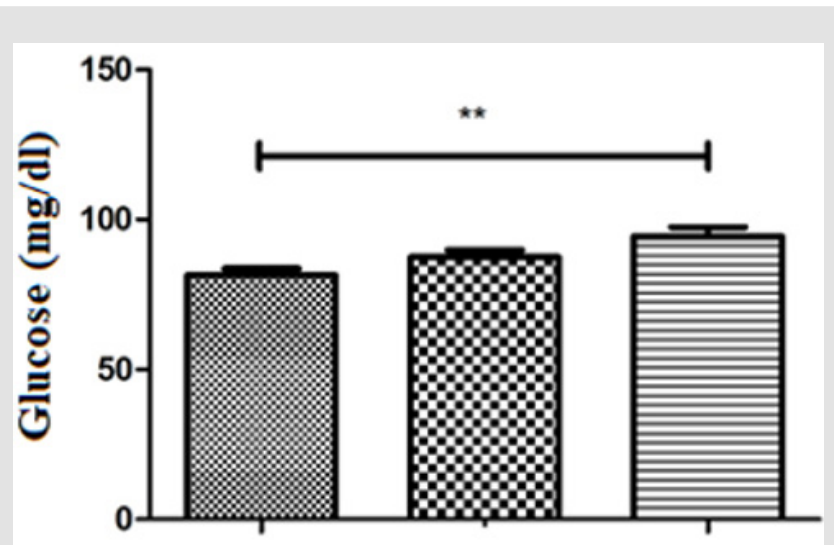

\section{Standard weight Overweight Obese}

Figure 1: Assessment of serum glucose levels $\left({ }^{* *} p \leq 0,01\right)$.

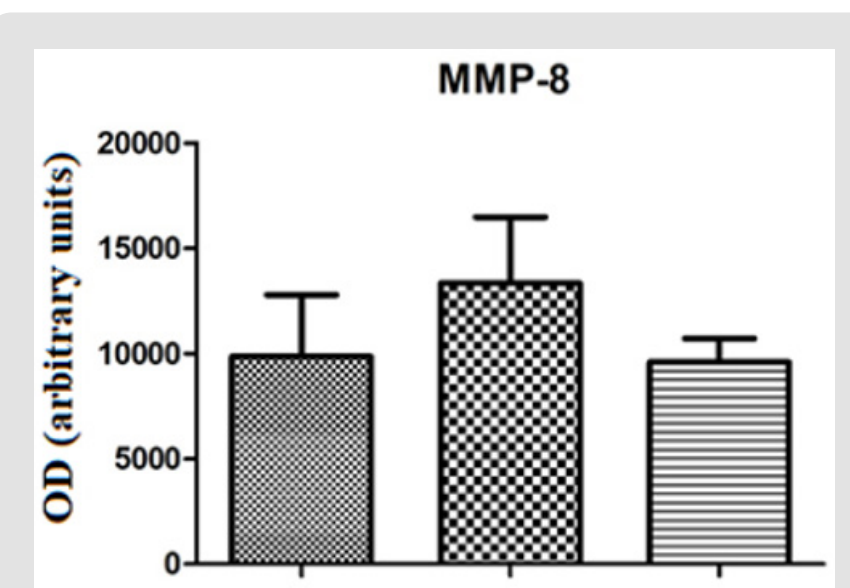

\section{Standard weight Overweight Obese}

Figure 2: Assessment of serum MMP-8 levels by slot blot.

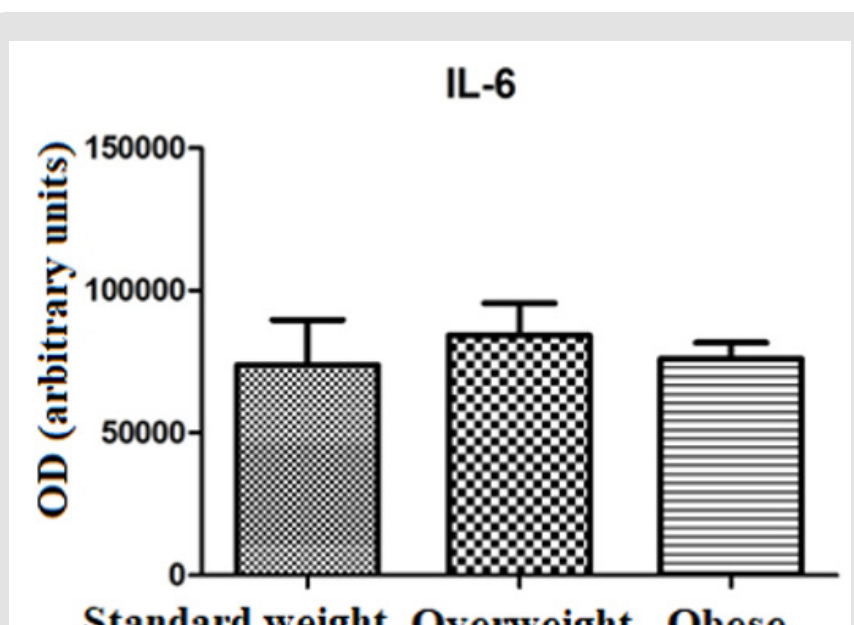

Figure 3: Assessment of serum IL-6 levels by slot blot.

The evaluation of TIMP-1 showed slightly higher levels in the group of standard weighted individuals (9960 \pm 4189$)$ and in the group of obese individuals ( $9235 \pm 3169$ ), in relation to the group of overweight individuals $(8374 \pm 4128)$, however the differences did not present statistical significance (Figure 4). The evaluation of the correlation between glucose levels, IL- 6 and the percentage of visceral fat (or android) displayed a moderate positive correlation between glucose levels and the percentage of visceral fat mass $(\mathrm{r}=$ 0,6112; $p \leq 0,0001$ ) (Figure 5). There was no statistically significant correlation between IL- 6 levels and the percentage of visceral fat mass ( $r=0,2887 ; p=0,0832$ ) (Figure 6). The evaluation of the correlation between the levels of MMP-8 and IL-6 presented a moderate positive correlation was found between MMP-8 and IL-6 serum levels ( $r=0,4510 ; p \leq 0.05)$, being the results statistically significant (Figure 7).

\section{TIMP-1}

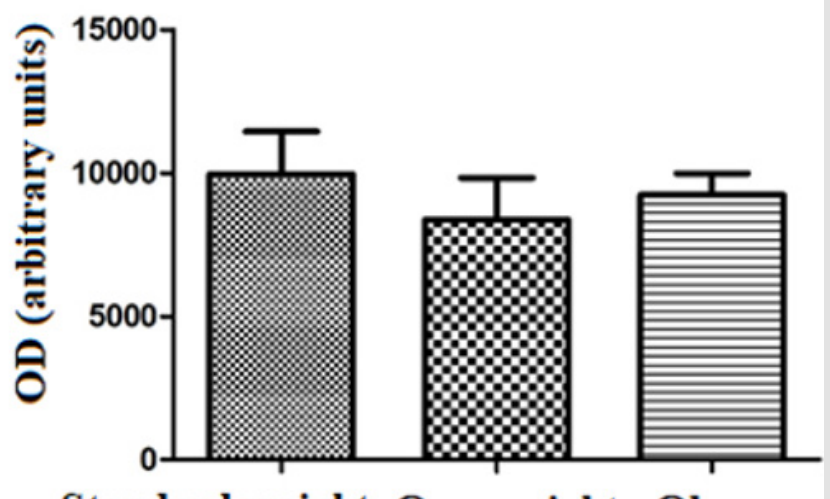

\section{Standard weight Overweight Obese}

Figure 4: Assessment of serum TIMP-1 levels by slot blot. 


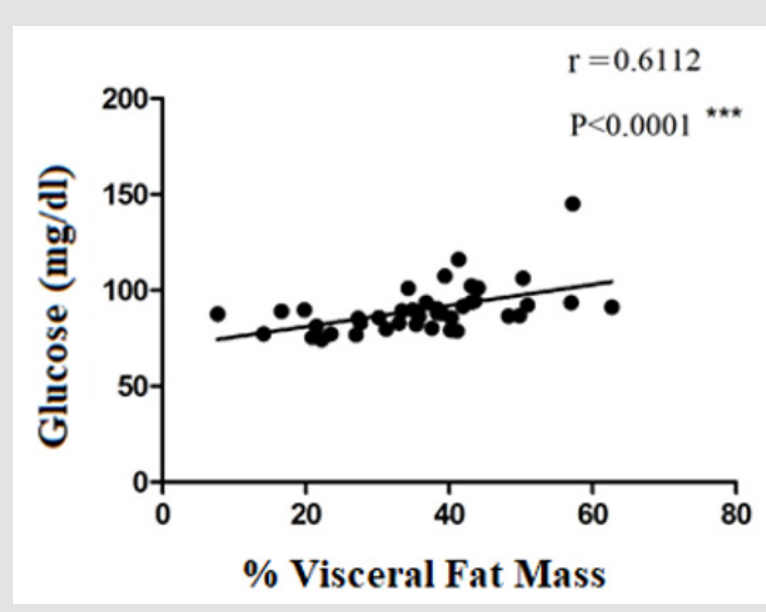

Figure 5: Analysis of blood glucose levels and visceral fat mass percentage correlation.

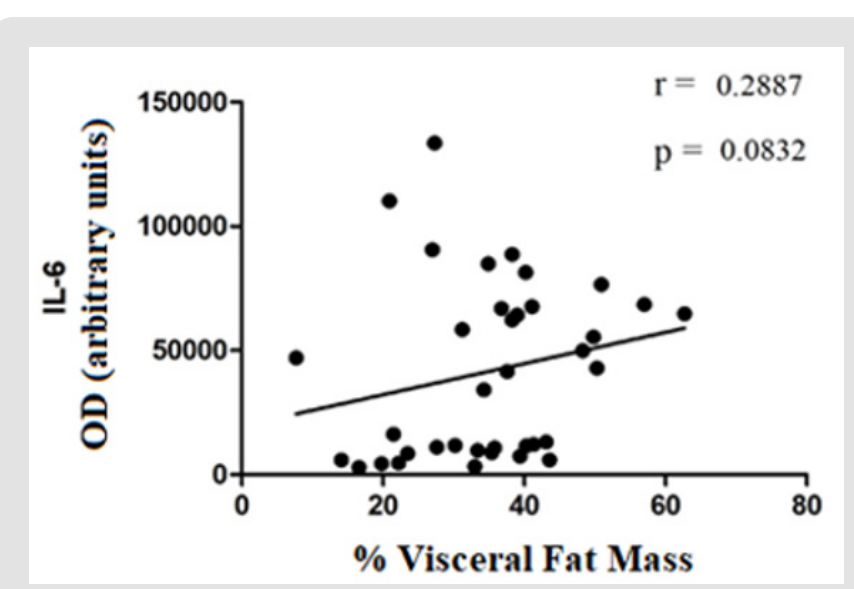

Figure 6: Analysis of IL-6 serum levels and visceral fat mass percentage correlation.

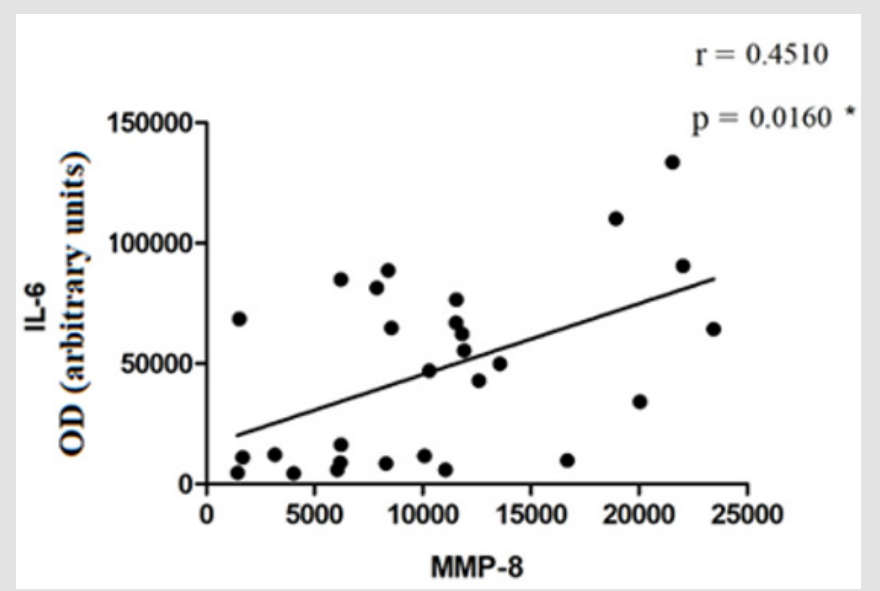

Figure 7: Analysis of serum MMP-8 and IL-6 levels correlation.

\section{Discussion}

Obesity is considered as a state of inflammation, in which adipose tissue and the liver are the main sources of cytokines and inflammatory proteins. This inflammation is local and is usually triggered and secondary to metabolic changes [18].
Expansion of adipose cells involves adipogenesis, angiogenesis and ECM remodeling. This remodeling is carried out by MMPs, which are regulated through gene expression levels, by activating proenzymes and by TIMPs [19]. MMPs, in addition to playing their role in the degradation of ECM, are also considered inflammation markers $[1,14]$. Regarding the study population, participants were grouped according to body fat mass percentage. The group of obese individuals had a higher percentage of fat mass compared to normal weighted individuals, this difference being statistically significant ( $p \leq 0,001)$. In relation to visceral fat mass percentage, the group of obese individuals had a higher percentage of visceral fat mass compared to individuals with excess weight, this difference being statistically significant $(p<0,05)$. This difference was even more significant between obese individuals and standard weighted individuals ( $\mathrm{p} \leq 0,001)$.

Although little is known about the biological effects of MMP-8, some studies have shown elevated levels of MMP-8 in atherosclerosis, childhood obesity and metabolic syndrome [20-22]. In the study by Andrade et al. lower serum MMP-8 levels were found in a population of obese women without comorbidities compared to the control group (19). Another study established evidence that, through its negative regulation, MMP-8 may have a protective or anti-inflammatory potential in human inflammatory diseases [23]. Lauhio et al. presented serum levels of MMP-8 significantly elevated in relation to standard weighted individuals [24]. In this study, MMP-8 levels were higher in the group of overweight individuals, compared to the group of standard weight and the group of obese individuals (Figure 2). These discrepant results suggest that there are different mechanisms of ECM remodeling that can be activated in different diseases [19]. Proinflammatory cytokines, such as IL-6, can influence adipocyte function, lipid metabolism, homeostasis, blood pressure and insulin sensitivity, therefore they play an important role in the development of diabetes, atherosclerosis and cardiovascular diseases [3]. Studies on obesity-associated inflammation are generally conducted in groups of older individuals, but there are some studies conducted in younger groups and adolescents that show similar results with high levels of proinflammatory cytokines, such as tumor necrosis factor alpha, IL-1 and IL-6 [5].

In this study, IL-6 levels were higher in the group of overweight individuals, in relation to the group of standard weighted individuals and to the group of obese individuals (Figure 3). MMPs activity is regulated by gene expression, by activating pro-enzymes and by endogenous inhibitors, TIMPs. In this study, TIMP-1 levels showed a slight decrease in the overweight group, compared to the standard weighted and obese individuals (Figure 4). TIMP-1 is described as an adipokine and the increase in its expression is related to obesity, so it was expected a significant increase in its expression in the group of obese individuals, which was not verified [25]. This contradiction shows that different mechanisms may be involved in the regulation of TIMP-1 expression [26]. Total adipose 
tissue and visceral adipose tissue have been shown to be associated differently with glucose-insulin metabolism in standard weight and obese individuals. While total adipose tissue is the best predictor of insulin sensitivity in normal weighted individuals, visceral adipose tissue is the parameter that correlates most strongly with the variables related to glucose-insulin metabolism in obese individuals [27]. A study of obese Caucasian and African women showed that visceral adipose tissue was the strongest predictor of changes in glucose metabolism [28].

The moderate positive correlation between serum glucose levels and visceral fat mass percentage $(r=0,6112 ; p \leq 0,0001)$ (Figure 5) is in line with the study by Ross et al., who showed an association between visceral fat mass and blood glucose [27]. Park et al. showed a significant correlation between IL-6 levels and visceral fat mass percentage [29]. In the study by Ghoski, et al. IL-6 mRNA expression in mouse models with polycystic ovary syndrome was increased in visceral adipose tissue compared to the control group [30]. In this study, a weak correlation was observed between IL-6 levels and visceral fat mass percentage $(r=0,2887$; $p=0,0832$ ) (Figure 6). These results may be related to the fact that adipose tissue is endocrine and produces numerous factors that contribute to systemic inflammation [29]. Gonçalves et al. showed high levels of MMP-8 in patients with metabolic syndrome, compared to the control group. This change was associated with increased concentrations of some inflammatory mediators, such as IL-6 [21]. In this study, a moderate positive correlation was observed between the serum levels of MMP-8 and the serum levels of IL-6 ( $\mathrm{r}=0,4510 ; \mathrm{p} \leq 0.05)$ (Figure 7). This may indicate that they can be stimulated by the same mechanisms in the inflammation process.

\section{Conclusion}

MMP-8 remains a little-known metalloproteinase and its results are not very clear. There seem to be different mechanisms of ECM remodelling involving MMP-8, which can be activated in different diseases. The correlation between IL-6 and visceral fat mass percentage may be due to the fact that the adipose tissue is endocrine and produces numerous factors that contribute to systemic inflammation. The moderate positive correlation between serum MMP-8 and IL-6 levels may indicate that they can be stimulated by the same mechanisms in the inflammation process. Despite the results obtained, further studies with a larger number of participants are needed. The fact that the total number of individuals studied is low may not show more tenuous relations between IL-6, MMP-8 and TIMP-1 in obesity.

\section{References}

1. Chavey C, Mari B, Monthouel MN, Bonnafous S, Anglard P, et al. (2003) Matrix metalloproteinases are differentially expressed in adipose tissue during obesity and modulate adipocyte differentiation. The Journal of biological chemistry 278(14): 11888-11896.

2. Vaittinen M, Kolehmainen M, Ryden M, Eskelinen M, Wabitsch M, et al. (2015) MFAP5 is related to obesity-associated adipose tissue and extracellular matrix remodeling and inflammation. Obesity (Silver Spring) 3(7): 1371-1378.

3. Paepegaey AC, Genser L, Bouillot JL, Oppert JM, Clement K, et al. (2015) High levels of CRP in morbid obesity: the central role of adipose tissue and lessons for clinical practice before and after bariatric surgery. Surgery for obesity and related diseases 11(1): 148-154.

4. Reilly SM, Saltiel AR (2017) Adapting to obesity with adipose tissue inflammation. Nature reviews Endocrinology 13(11): 633-643.

5. Mehmood ZH, Papandreou D (2016) An Updated Mini Review of Vitamin D and Obesity: Adipogenesis and Inflammation State. Open access Macedonian journal of medical sciences 4(3): 526-532.

6. Zeyda M, Stulnig TM (2009) Obesity, inflammation, and insulin resistance--a mini-review. Gerontology 55(4): 379-386.

7. Xu E, Pereira MMA, Karakasilioti I, Theurich S, Al Maarri M, et al. (2017) Temporal and tissue-specific requirements for T-lymphocyte IL-6 signalling in obesity-associated inflammation and insulin resistance. Nature communications 8: 14803.

8. Jorge ASB, Andrade JMO, Paraiso AF, Jorge GCB, Silveira CM, et al. (2018) Body mass index and the visceral adipose tissue expression of IL-6 and TNF-alpha are associated with the morphological severity of nonalcoholic fatty liver disease in individuals with class III obesity. Obesity research \& clinical practice 12(Supply 2): 1-8.

9. Boeta Lopez K, Duran J, Elizondo D, Gonzales E, Rentfro A, et al. (2018) Association of interleukin-6 polymorphisms with obesity or metabolic traits in young Mexican Americans. Obesity science \& practice 4(1): 8596.

10. Chen F, Chen D, Zhao X, Yang S, Li Z, et al. (2017) Interleukin-6 deficiency facilitates myocardial dysfunction during high fat diet-induced obesity by promoting lipotoxicity and inflammation. Biochimica et biophysica acta 1863(12): 3128-3141.

11. Peeters SA, Engelen L, Buijs J, Chaturvedi N, Fuller JH, et al. (2015) Plasma levels of matrix metalloproteinase-2, $-3,-10$, and tissue inhibitor of metalloproteinase-1 are associated with vascular complications in patients with type 1 diabetes: the EURODIAB Prospective Complications Study. Cardiovascular diabetology 14: 31.

12. Maquoi E, Munaut C, Colige A, Collen D, Lijnen HR (2002) Modulation of adipose tissue expression of murine matrix metalloproteinases and their tissue inhibitors with obesity. Diabetes 51(4): 1093-1101.

13. Dejonckheere E, Vandenbroucke RE, Libert C (2011) Matrix metalloproteinase 8 has a central role in inflammatory disorders and cancer progression. Cytokine \& growth factor reviews 22(2): 73-81.

14. Franco C, Patricia HR, Timo S, Claudia B, Marcela H (2017) Matrix Metalloproteinases as Regulators of Periodontal Inflammation. International journal of molecular sciences 18(2).

15. Gonzalez Lopez A, Aguirre A, Lopez Alonso I, Amado L, Astudillo A, et al. (2012) MMP-8 deficiency increases TLR/RAGE ligands S100A8 and S100A9 and exacerbates lung inflammation during endotoxemia. PloS one $7(6)$ : e39940.

16. Gallagher D, Heymsfield SB, Heo M, Jebb SA, Murgatroyd PR, et al. (2000) Healthy percentage body fat ranges: an approach for developing guidelines based on body mass index. The American journal of clinical nutrition 72(3): 694-701.

17. Caseiro A, Vitorino R, Barros AS, Ferreira R, Calheiros Lobo MJ, et al. (2012) Salivary peptidome in type 1 diabetes mellitus. Biomedical chromatography. BMC 26(5): 571-582.

18. Rubio Jurado B, Balderas Pena LM, Garcia Luna EE, Zavala Cerna MG, Riebeling Navarro C, et al. (2018) Obesity, Thrombotic Risk, and Inflammation in Cancer. Advances in clinical chemistry 85: 71-89.

19. Andrade VL, Petruceli E, Belo VA, Andrade Fernandes CM, Caetano Russi CV, et al. (2012) Evaluation of plasmatic MMP-8, MMP-9, TIMP-1 and MPO levels in obese and lean women. Clinical biochemistry 45(6): 412415 . 
20. Belo VA, Souza Costa DC, Lana CM, Caputo FL, Marcaccini AM, et al. (2009) Assessment of matrix metalloproteinase (MMP)-2, MMP-8, MMP-9, and their inhibitors, the tissue inhibitors of metalloproteinase (TIMP)-1 and TIMP-2 in obese children and adolescents. Clinical biochemistry $42(10$ 11): $984-990$

21. Goncalves FM, Jacob Ferreira AL, Gomes VA, Casella Filho A, Chagas AC, et al. (2009) Increased circulating levels of matrix metalloproteinase (MMP)-8, MMP-9, and pro-inflammatory markers in patients with metabolic syndrome. Clinica chimica acta 403(1-2): 173-177.

22. LengletS, Mach F, Montecucco F (2013) Role of matrix metalloproteinase-8 in atherosclerosis. Mediators of inflammation 2013: 659282.

23. Saarinen R, Pitkaranta A, Kolho KL, Tervahartiala T, Sorsa T, et al. (2016) Decreased salivary matrix metalloproteinase-8 reflecting a defensive potential in juvenile parotitis. International journal of pediatric otorhinolaryngology 80: 74-77.

24. Lauhio A, Farkkila E, Pietilainen KH, Astrom P, Winkelmann A, et al. (2016) Association of MMP-8 with obesity, smoking and insulin resistance. European journal of clinical investigation 46(9): 757-765.

25. Meissburger B, Stachorski L, Roder E, Rudofsky G, Wolfrum C (2011) Tissue inhibitor of matrix metalloproteinase 1 (TIMP1) controls adipogenesis in obesity in mice and in humans. Diabetologia 54(6): 1468-1479.

26. Orbe J, Fernandez L, Rodriguez JA, Rabago G, Belzunce M, et al. (2003) Different expression of MMPs/TIMP-1 in human atherosclerotic lesions. Relation to plaque features and vascular bed. Atherosclerosis 170(2): 269-276.

27. Ross R, Fortier L, Hudson R (1996) Separate associations between visceral and subcutaneous adipose tissue distribution, insulin and glucose levels in obese women. Diabetes care 19(12): 1404-1411.

28. Bi X, Seabolt L, Shibao C, Buchowski M, Kang H, et al. (2015) DXAmeasured visceral adipose tissue predicts impaired glucose tolerance and metabolic syndrome in obese Caucasian and African American women. European journal of clinical nutrition 69(3): 329-336.

29. Park HS, Park JY, Yu R (2005) Relationship of obesity and visceral adiposity with serum concentrations of CRP, TNF-alpha and IL-6. Diabetes research and clinical practice 69(1): 29-35.

30. Ghowsi M, Khazali H, Sisakhtnezhad S (2018) Evaluation of TNF-alpha and IL-6 mRNAs expressions in visceral and subcutaneous adipose tissues of polycystic ovarian rats and effects of resveratrol. Iranian journal of basic medical sciences 21(2): 165-174.
ISSN: 2574-1241

DOI: $10.26717 /$ BJSTR.2020.25.004229

Armando Caseiro. Biomed J Sci \& Tech Res

(C) This work is licensed under Creative

Submission Link: https://biomedres.us/submit-manuscript.php

$\begin{array}{ll}\text { BIOMEDICAL } & \text { Assets of Publishing with us } \\ \text { RESEARCHES } & \text { - Global archiving of articles } \\ \text { - Immediate, unrestricted online access }\end{array}$

